\title{
PERDA COGNITIVA E DEPENDÊNCIA FUNCIONAL EM IDOSOS LONGEVOS RESIDENTES EM INSTITUIÇÕES DE LONGA PERMANÊNCIA*
}

\author{
Renato Novaes Chaves ${ }^{1}$, Pollyanna Viana Lima², Tatiane Dias Casimiro Valença ${ }^{3}$, Elaine dos Santos Santana ${ }^{4}$, \\ Maykon dos Santos Marinho ${ }^{5}$ Luciana Araújo dos Reis ${ }^{6}$
}

\begin{abstract}
RESUMO: O objetivo foi avaliar o grau de dependência funcional quanto às Atividades Básicas da Vida Diária e a perda cognitiva de idosos longevos residentes em duas instituições de longa permanência para idosos do interior do Estado da Bahia. Pesquisa do tipo quantitativa, transversal, realizada no ano de 2016, com 20 idosos longevos. Avaliada a cognição do entrevistado, por meio do Mini Exame do Estado Mental, a dependência funcional quanto às Atividades Básicas da Vida Diária por meio do Índice de Barthel e da Escala de Katz, e o perfil sociodemográfico. Os resultados apontaram a prevalência da faixa etária entre 80 a 85 anos (90,0\%). O sexo feminino apresentou maior porcentagem de perda cognitiva $(28,5 \%)$ seguido dos não alfabetizados, homens e mulheres, $(37,3 \%)$. O estudo subsidia a discussão a partir do impacto que a perda cognitiva e a dependência funcional trazem para a saúde do idoso longevo.
\end{abstract}

DESCRITORES: Cognição; Longevidade; Envelhecimento; Instituição de Longa Permanência para Idosos.

\section{COGNITIVE LOSS AND FUNCTIONAL DEPENDENCE IN LONG-LIVED ELDERLY IN HOMES FOR THE AGED}

ABSTRACT: The objective was to assess the degree of functional dependence for the Basic Activities of Daily Living and the cognitive loss of long-lived elderly at two long-term care institutions for the elderly in the State of Bahia. Quantitative, cross-sectional research developed in 2016, involving 20 long-lived elderly. The interviewee's cognition was assessed by means of the Mini-Mental State Examination, the functional dependence for the Basic Activities of Daily Living by means of the Barthel Index and the Katz Scale, and the sociodemographic profile. The results appointed the prevalence of the age range between 80 and 85 years $(90.0 \%)$. The female sex presented a higher percentage of cognitive loss (28.5\%), followed by illiterate men and women, $(37.3 \%)$. The study supports the discussion based on the impact of the cognitive loss and the functional dependence for the health of the long-lived elderly.

DESCRIPTORS: Cognition; Longevity; Aging; Homes for the Aged.

\section{PÉRDIDA COGNITIVA Y DEPENDENCIA FUNCIONAL EN ANCIANOS LONGEVOS RESIDENTES EN HOGARES PARA ANCIANOS}

RESUMEN: La finalidad fue evaluar el grado de dependencia funcional para las Actividades Básicas de la Vida Diaria y la pérdida cognitiva de ancianos longevos residentes en dos hogares para ancianos del interior del Estado de Bahia. Investigación cuantitativa, trasversal, desarrollada en 2016 con 20 ancianos longevos. Fue evaluada la cognición del entrevistado mediante el Mini Examen del Estado Mental, la dependencia funcional para las Actividades Básicas de la Vida Diaria mediante el Índice de Barthel y la Escala de Katz, y el perfil sociodemográfico. Los resultados apuntaron la prevalencia del rango de edad entre 80 a 85 años (90,0\%). El sexo femenino presentó mayor porcentaje de pérdida cognitiva (28,5\%), seguido de los no alfabetizados, hombres y mujeres, (37,3\%). El estudio subsidia la discusión a partir del impacto de la pérdida cognitiva y la dependencia funcional para la salud del anciano longevo. DESCRIPTORES: Cognición; Longevidad; Envejecimiento; Hogares para Ancianos. *Artigo extraído da dissertação intitulada: "Representações Sociais e Memórias de idosos longevos sobre o processo de
envelhecimento e a dependência funcional". Universidade Estadual do Sudoeste da Bahia, 2017.

${ }^{1}$ Enfermeiro. Mestrando em Memória: Linguagem e Sociedade. Universidade Estadual do Sudoeste da Bahia. Vitória da Conquista, BA, Brasil.

${ }^{2}$ Enfermeira. Doutoranda em Memória: Linguagem e Sociedade. Universidade Estadual do Sudoeste da Bahia. Vitória da Conquista, BA, Brasil.

${ }^{3}$ Fisioterapeuta. Doutoranda em Memória: Linguagem e Sociedade. Universidade Estadual do Sudoeste da Bahia. Vitória da Conquista, BA, Brasil.

${ }^{4}$ Enfermeira. Mestranda do Programa de Pós-Graduação em Memória: Linguagem e Sociedade - PPGMLS da Universidade Estadual do Sudoeste da Bahia. Vitória da Conquista, BA, Brasil.

${ }^{5}$ Enfermeiro. Doutorando em Memória: Linguagem e Sociedade. Universidade Estadual do Sudoeste da Bahia. Vitória da Conquista, BA, Brasil.

${ }^{6}$ Fisioterapeuta. Doutora em Ciências da Saúde. Docente de Fisioterapia e do Programa de Pós-Graduação em Memória: Linguagem e Sociedade da Universidade Estadual do Sudoeste da Bahia. Vitória da Conquista, BA, Brasil.

Autor Correspondente:

Recebido: $16 / 09 / 2016$

Renato Novaes Chaves

Finalizado: 08/02/2017

Universidade Estadual do Sudoeste da Bahia

Rua G, 332 - 45.026.720 - Vitória da Conquista, BA, Brasil

E-mail: re.novaes81@icloud.com 


\section{- INTRODUÇÃO}

No Brasil, dos mais de 206 milhões de habitantes, quase 1,7\% são idosos longevos, aqueles que têm idade igual ou maior que 80 anos. A projeção para o ano de 2030 é de que chegue a quase $3 \%$ de longevos em todo o país ${ }^{(1)}$. O processo de envelhecimento foi percebido em várias regiões do país, principalmente no período de 1970 a $2010^{(2)}$.

Dessa forma, as mudanças na estrutura etária da população brasileira evidencia um país que deixa de ter uma população meramente jovem, uma vez que a idade média dos indivíduos passou de 18 para 27 anos no mesmo período ${ }^{(3)}$.

Sendo assim, envelhecer significa ter a certeza de que diversas modificações ocorrerão. E, uma das principais alterações que os idosos vivenciam passa pelo campo da capacidade funcional. Esta é entendida como a habilidade que a pessoa possui em efetuar determinada ação ou tarefa de modo independente ${ }^{(4)}$, e é um excelente indicador da funcionalidade em idosos ${ }^{(5)}$.

O declínio da capacidade funcional do idoso está relacionado com o próprio processo de envelhecimento, que tem relação com o comprometimento das funções gerais e o surgimento de patologias que afetam a funcionalidade, contribuindo para a dependência funcional ${ }^{(6)}$, que significa restrição ou ajuda na participação das atividades do dia a dia.

Ademais, cabe ressaltar que também podem surgir, no processo de envelhecimento, alterações de saúde associadas à diminuição da função cognitiva( ${ }^{(7)}$. Esta é caracterizada pela dificuldade em manter a memória recente ou de evocação que vai muito além do que é relacionado com o envelhecimento normal, podendo indicar, em quadros mais severos, casos de demência ou doença de Alzheimer ${ }^{(8-9)}$.

Dessa forma, mesmo as alterações tidas como normais são de suma importância para compreender os processos envolvidos na perda cognitiva, pois podem afetar as atividades diárias do idoso e ainda contribuir para a distinção do que é normal ou patológico a partir de estados de doença ${ }^{(7)}$. Sendo assim, o declínio das funções motoras e cognitivas repercute tanto nas Atividades Básicas da Vida Diária (ABVD) como nas instrumentais ${ }^{(10)}$.

Também é possível salientar que avaliação do comprometimento funcional, em idosos institucionalizados, é um excelente marcador para o envelhecimento, pois estão relacionados à dependência e também ao maior risco de quedas ${ }^{(10)}$.

Desse modo, optou-se por pesquisar idosos longevos residentes em Instituição de Longa Permanência para Idosos (ILPI), pois, devido à dependência funcional, déficit cognitivo, abandono e/ ou outros fatores, alguns idosos são submetidos ao internamento em ILPI ou casas de repouso como única opção de moradia.

As ILPI apresentam uma dualidade. Apesar de assistirem de modo integral e prestarem cuidado, também proporcionam o isolamento, a inatividade física e social, e o distanciamento da família(11). Elas recebem diferentes nomenclaturas, como asilos, casas geriátricas, abrigos, casas de repouso, entre outras. No entanto, independente da terminologia, são destinadas a receber indivíduos a partir dos 60 anos de idade em regime de internamento ${ }^{(12)}$.

A justificativa para o estudo se pauta na capacidade científica de trabalhar com um tema que exige conhecimento aprofundado e sistemático, e a possibilidade de abordar uma temática de grande relevância social, a qual a família e a sociedade não costumam considerar.

A identificação precoce da dependência funcional e da perda cognitiva pode contribuir para melhorar a qualidade de vida do idoso, bem como o processo de envelhecimento. A partir daí, pode-se traçar um panorama dos aspectos funcionais e cognitivos dos longevos, para que possam ser implementadas ações de reabilitação e promoção da saúde para esses idosos. Dessa forma, a dependência funcional e a perda cognitiva em idosos estão associadas a uma série/conjunto de fatores que determinam a capacidade que o idoso possui em se manter independente nas suas ABVD.

Nesta perspectiva, o presente estudo tem por objetivo avaliar o grau de dependência funcional quanto às ABVD e à cognição de idosos longevos residentes em duas ILPI do interior do Estado da 
Trata-se de pesquisa com abordagem quantitativa, de corte transversal. Realizada em duas ILPI, localizadas nos municípios de Vitória da Conquista e Itapetinga, ambas situadas no sudoeste do Estado da Bahia. Estes locais foram escolhidos com base nos seguintes critérios: possuir idosos longevos e permitir autorização da ILPI para coleta dos dados. São instituições filantrópicas, que se mantêmpor meio da própria aposentadoria dos idosos, mas também recebem doações da sociedade, município, empresas e organizações privadas.

Os participantes foram os idosos longevos residentes nas duas ILPI perfazendo uma população de 35 longevos. Os critérios de inclusão adotados foram: possuir 80 anos de idade ou mais, pois quanto mais longevo maiores são as chances de surgir dependência funcional ou função cognitiva diminuída ${ }^{(13)}$; Apresentar algum tipo de comprometimento funcional de acordo com o Índice de Barthel e Escala de Katz, pois quanto maior o grau de dependência funcional, mais cuidados nas ABVD serão requeridas para o idoso. A dependência associada com a perda cognitiva interfere na qualidade de vida do idoso dentro da ILPI.

Foram excluídos os longevos com alguma patologia neurodegenerativa que afete a função cognitiva, sendo verificado por meio do diagnóstico médico contido nos prontuários. Os idosos não dependentes foram excluídos, pois não estão dentro dos objetivos da pesquisa.

A amostra foi composta por 20 idosos longevos, sendo 10 homens e 10 mulheres, selecionados conforme os critérios de elegibilidade. Foram sete longevos portadores de Alzheimer, e oito estavam com sequelas de Acidente Vascular Cerebral (AVC) que afetaram a fala, inviabilizando a entrevista.

A coleta dos dados ocorreu entre os dias 04 a 08 de janeiro de 2016 na ILPI de Vitória da Conquista BA e nos dias 11 a 15 de janeiro de 2016 na ILPI de Itapetinga - BA, no período matutino das 10h00min às $12 \mathrm{~h} 00 \mathrm{~min}$ e vespertino das $15 \mathrm{~h} 00 \mathrm{~min}$ as $17 \mathrm{~h} 00 \mathrm{~min}$. $\mathrm{E}$, para que houvesse um clima de cordialidade e confiança, a coleta aconteceu no quarto dos longevos e se deu como um bate papo entre os pesquisadores e os idosos. Não houve presença de terceiros no ato da entrevista. Os procedimentos para coleta dos dados obedeceram às seguintes etapas:

Etapa 1 - Avaliação Cognitiva por meio do Mini Exame do Estado Mental (MEEM)(14): teste rápido, composto por 11 itens de fácil aplicação que avalia sete funções cognitivas: Orientação Temporal (5 pontos), Orientação Espacial (5 pontos), Registro - de três palavras (3 pontos), Atenção e Cálculo (5 pontos), Memória e Evocação (3 pontos), e Linguagem (9 pontos). O escore total do MEEM varia de 0 a 30 pontos, e deve-se levar em consideração a escolaridade do idoso. Para somatória dos pontos, a nota de corte foi: "Resultados: Pontuação total $=30$ pontos. As notas de corte sugeridas são: Analfabetos = 19. De 1 a 3 anos de escolaridade $=23$. De 4 a 7 anos de escolaridade $=24 .>7$ anos de escolaridade $=$ $28^{\prime \prime}(14: 138)$.

Etapa 2 - Avaliação do perfil sociodemográfico: perguntas para estabelecer o perfil dos participantes, tais como: idade (80 a 85 anos, acima de 85 anos), sexo (masculino e feminino), escolaridade (Alfabetizados - de 1 a 3 anos de estudo, de 4 a 7 anos de estudo e >7 anos de estudo; não alfabetizados), profissão antes de aposentar (respondido sem opções preestabelecidas), estado civil (Solteiros ou Separados, União estável, Viúvos).

Etapa 3 - Avaliação da Funcionalidade: foram usados dois instrumentos nesta etapa, o Índice de Barthel(14) que mede o grau de assistência exigido para um indivíduo, sendo composto por 10 itens de ABVD envolvendo alimentação, higiene pessoal, uso dos sanitários, banho, vestuário, controle de esfíncteres (vesical e intestinal), deambulação ou cadeira de rodas, transferência da cadeira para a cama, subir escadas. A pontuação varia de acordo com os pontos obtidos, sendo 100 pontos independentes; de 99 a 76 pontos tem dependência leve; de 75 a 51 dependência moderada; de 50 a 26 dependência severa; Menos que 25 pontos é totalmente dependente. O outro instrumento foi a Escala de Katz(14) que avalia 06 categorias das ABVD, tais como banho, ir ao banheiro, vestuário, continências, transferências e alimentação. A classificação de 5 a 6 pontos é considerado independente; 3 a 4 pontos tem dependência moderada; 2 ou menos pontos indica que o idoso é muito dependente.

A partir dos dados, procedeu-se a tabulação dos resultados com o auxílio do programa Statistical 
Package for Social Sciences (SPSS ${ }^{\circledR}$ ), versão 20.0 no qual foi realizada a análise estatística descritiva por meio da média, desvio padrão e frequência absoluta, sendo usadas tabelas para melhor visualização das análises.

A pesquisa foi aprovada com o parecer $n^{\circ} 1.333 .766$ pelo Comitê de Ética em Pesquisas da Universidade Estadual do Sudoeste da Bahia.

\section{RESULTADOS}

Com base na Tabela 1, pode-se traçar o perfil dos longevos. A idade prevalente foi de 80 a 85 anos $18(90 \%)$, com relação à profissão antes de aposentar eram lavradores 9 (45\%), seguidos de serviços domésticos 7 (35\%). Quanto à escolaridade, 14 (70\%) é alfabetizada, no entanto, com apenas um a três anos de estudo 10 (50\%), o que configura baixa escolaridade, e há um predomínio de solteiros ou separados 11 (55\%). Pode-se observar também que a profissão antes de aposentar e a escolaridade determinam que esses idosos provem de uma situação de baixo nível socioeconômico.

Tabela 1 - Caracterização Sociodemográfica dos idosos residentes nas ILPI. Vitória da Conquista e Itapetinga, BA, Brasil, 2016

\begin{tabular}{|c|c|c|c|}
\hline \multicolumn{2}{|c|}{ Variáveis do estudo } & Quantitativo & Percentual \\
\hline \multicolumn{4}{|l|}{ Faixa etária } \\
\hline \multicolumn{2}{|l|}{80 a 85 anos } & 18 & 90 \\
\hline \multicolumn{2}{|c|}{ Acima de 85 anos } & 2 & 10 \\
\hline \multicolumn{4}{|c|}{ Profissão antes de aposentar } \\
\hline \multicolumn{2}{|c|}{ Serviço doméstico } & 7 & 35 \\
\hline \multicolumn{2}{|c|}{ Lavrador (a) } & 9 & 45 \\
\hline \multicolumn{2}{|l|}{ Caminhoneiro } & 1 & 5 \\
\hline \multicolumn{2}{|l|}{ Pintor } & 1 & 5 \\
\hline \multicolumn{2}{|l|}{ Pedreiro } & 1 & 5 \\
\hline \multicolumn{2}{|c|}{ Funcionário Público } & 1 & 5 \\
\hline \multicolumn{4}{|l|}{ Escolaridade } \\
\hline \multicolumn{2}{|c|}{ Não Alfabetizados } & 6 & 30 \\
\hline \multirow[t]{3}{*}{ Alfabetizados } & De 1 a 3 anos de estudo & 10 & 50 \\
\hline & De 4 a 7 anos de estudo & 3 & 15 \\
\hline & Mais de 7 anos de estudo & 1 & 5 \\
\hline \multicolumn{4}{|l|}{ Estado civil } \\
\hline \multicolumn{2}{|c|}{ Solteiro (a) / Separado (a) } & 11 & 55 \\
\hline \multicolumn{2}{|l|}{ União Estável } & 1 & 5 \\
\hline \multicolumn{2}{|l|}{ Viúvo (a) } & 8 & 40 \\
\hline \multicolumn{2}{|l|}{ Total } & 20 & 100 \\
\hline
\end{tabular}

A Tabela 2 apresenta o percentual dos longevos de acordo com a classificação nas ABVD. É possível constatar que todos os longevos pesquisados apresentaram graus diferenciados de dependência funcional. No entanto, nas ABVD avaliadas pelo Índice de Barthel há uma predominância de dependência leve 8 (40\%) seguido de dependência moderada 5 (25\%), enquanto que no Escala de Katz, 13 (65\%) apresentam dependência moderada, seguida de muito dependentes 7 (35\%). 
Tabela 2 - Distribuição dos idosos longevos residentes nas ILPI sobre a dependência funcional. Vitória da Conquista e Itapetinga, BA, Brasil, 2016

\begin{tabular}{lcc} 
Dependência Funcional & Quantitativo & Percentual \\
\hline Índice de Barthel & & \\
\hline ( $\leq 25)$ Totalmente Dependente & 3 & 15 \\
\hline (26 a 50) Dependência Severa & 4 & 20 \\
\hline (51 a 75) Dependência Moderada & 5 & 25 \\
\hline (76 a 99) Dependência Leve & 8 & 40 \\
\hline Escala de Katz & & \\
\hline ( $\leq 2)$ Muito Dependente & 7 & 35 \\
\hline (4) Dependência Moderada & 13 & 65 \\
\hline Total & 20 & 100
\end{tabular}

A Tabela 3 apresenta o perfil cognitivo dos idosos obtido por meio dos cinco domínios de avaliação do MEEM. É possível constatar que apenas no domínio "registro" 20 (100\%) atingiu a média, na "orientação", "atenção e calculo" 13 (65\%) estão abaixo da média, na "linguagem" observa-se que 14 (70\%), o maior percentual, não conseguiu alcançar a média, já na "memória e evocação" foi o domínio em que 11 (55\%) ficaram abaixo da média.

Tabela 3 - Perfil Cognitivo dos idosos longevos residentes em ILPI. Vitória da Conquista e Itapetinga, BA, Brasil, 2016

\begin{tabular}{lcccc} 
Domínios do MEEM & $\begin{array}{c}\text { Média total } \\
\text { (DP) }\end{array}$ & $\begin{array}{c}\text { Acima da } \\
\text { média (\%) }\end{array}$ & $\begin{array}{c}\text { Abaixo da } \\
\text { média (\%) }\end{array}$ & PMin. \\
\hline Orientação (PM =10) & $7,05(1,276)$ & 25 & 65 & 5 \\
\hline Registro (PM =3) & $3,00(0)$ & 100 & 0 & 3 \\
\hline Atenção e cálculo (PM = 5) & $2,20(2,19)$ & 25 & 65 & 0 \\
\hline Memória e Evocação (PM =3) & $2,25(0,851)$ & 45 & 55 & 0 \\
\hline Linguagem (PM =9) & $7,15(1,30)$ & 30 & 70 & 5
\end{tabular}

PM= Ponto Máximo. DP= Desvio Padrão. PMin= Ponto Mínimo.

Já a Tabela 4 apresenta a perda cognitiva dos longevos do sexo masculino e feminino levando-se em consideração a somatória final das pontuações de cada participantena função cognitiva do MEEM, a partir da escolaridade proposta pelo Ministério da Saúde ${ }^{(14)}$.

Tabela 4 - Perda cognitiva dos idosos longevos residentes em ILPI. Vitória da Conquista e Itapetinga, BA, Brasil, 2016

\begin{tabular}{llcccc} 
Variáveis & & $\mathbf{n}$ & $\mathbf{M}$ & Min - Max & PC (\%) \\
\hline Sexo & Escolaridade & & & & \\
\hline \multirow{2}{*}{ Masculino } & Alfabetizados* & 7 & 23 & $19-28$ & 23,3 \\
\cline { 2 - 6 } & Não Alfabetizados & 3 & 19,3 & $18-21$ & 35,7 \\
\hline \multirow{2}{*}{ Feminino } & Alfabetizados* & 7 & 22,8 & $18-28$ & 23,7 \\
\cline { 2 - 6 } & Não Alfabetizados & 3 & 18,6 & $18-20$ & 37,8
\end{tabular}

$\mathrm{n}=$ Amostra. $\mathrm{M}=$ Média de pontos. Min = Mínimo. Max = Máximo. $\mathrm{PC}=$ Perda Cognitiva.

*De 1 a 3 anos de estudo, de 4 a 7 anos de estudo e $>7$ anos de estudo. 
Sendo assim, é possível traçar um perfil de déficit cognitivo nos pesquisados, onde se observa que as melhores médias foram alcançadas pelos longevos alfabetizados tanto masculinos como femininos, com 23 e 22,8 pontos respectivamente. E a perda cognitiva foi maior entre os não alfabetizados, sendo $35,7 \%$ para o sexo masculino e $37,8 \%$ para o feminino.

Dessa forma, pode-se inferir que sexo feminino apresentou maior porcentagem de perda cognitiva, quando comparado com o masculino, na mesma escolaridade. O que indica que os idosos longevos com baixa escolaridade apresentaram maior perda cognitiva, bem como médias mais baixas na pontuação geral.

\section{DISCUSSÃO}

O perfil dos idosos longevos permite conhecer e entender a sua caracterização social e a partir disso traçar paralelos de como eles desenvolveram a dependência funcional. Também pode contribuir para analisar o modo como envelheceram, pois nota-se, a partir das profissões que exerciam na sua juventude, que trabalhavam com serviços braçais, que demandavam muita energia e desgaste físico.

Desse modo, uma recente pesquisa ${ }^{(15)}$ que investigou as consequências que o trabalho acarreta, ao longo da vida, para as alterações posturais e problemas de coluna de idosos participantes de um grupo de convivência na cidade de Município de Teutônia, Estado do Rio Grande do Sul, constatou que o desgaste físico ocasionado pelo trabalho pode afetar a qualidade de vida dos idosos.

Ainda para os autores ${ }^{(15)}$, os idosos participantes do estudo relacionaram as alterações posturais com a atividade laboral ao longo dos anos, uma vez que eles trabalhavam em atividades braçais na zona rural. Sendo assim, pode-se inferir que os longevos pesquisados neste estudo também tiveram trabalhos braçais ao longo da vida, o que pode ter contribuído para a dependência funcional.

Ademais, estar em uma ILPI pode ser considerado condição para que quadros depressivos se instalem, no entanto, se observarmos o estado civil e o relacionarmos com a solidão vivenciada na ILPI, pois apenas $1(5 \%)$ tinha união estável, poderemos dizer que a falta de um companheiro(a) aumenta as chances dos idosos desenvolverem tal quadro. Nesse sentido, um estudo ${ }^{(16)}$ sobre depressão em idosos aponta que a solidão juntamente com o isolamento social são as causas principais de admissões nas ILPI.

Com relação à dependência funcional, observamos que os idosos têm graus variados de comprometimento funcional no que tange às $A B V D$, requerendo maiores cuidados por parte da equipe dentro das ILPI pesquisadas. Nesse sentido, um estudo ${ }^{(13)}$ realizado com idosos residentes em ILPI no Estado de Minas Gerais constatou que 73 (58,4\%) deles apresentaram algum grau de dependência de acordo com a Escala de Katz, e que quando comparada a idade com o grau de dependência, este estudo mostrou que a dependência aumentava com o avançar da idade.

Nesse sentido, outro estudo(17) corrobora com esses resultados e afirma que, à medida que se envelhece, o idoso fica mais vulnerável à ação progressiva do meio ambiente, com maior dificuldade para as tarefas diárias, o que pode contribuir para uma diminuição de capacidade funcional.

A esse respeito, um recente estudo ${ }^{(18)}$ chama atenção de que, além das doenças crônicas que podem comprometer a capacidade funcional do idoso, o processo de envelhecimento também pode contribuir para este comprometimento, sendo, portanto, chamado de envelhecimento funcional.

No entanto, percebe-se que "com o passar dos anos fica mais evidente com a chegada da velhice, que o corpo humano sofre um declínio fisiológico comprometendo a sua capacidade funcional" (19:68). Dessa forma, nem todos os idosos apresentam quadros de comprometimento, e quando os tem podem ter graus diferenciados.

Portanto, podemos inferir que a velhice é uma construção por meio dos qual os indivíduos, nas suas diversas redes sociais, se constituem, seja no meio familiar, social, cultural, entre outros. Sendo assim, deve-se entender que envelhecer pode ser algo diferenciado para cada indivíduo.

Nesse contexto, ao pensar em dependência funcional de idosos que residem em ILPI, é importante 
levar em consideração as ações que possam minimizá-la, proporcionando mais autonomia ao idoso e, também, melhor condição de intervenção para a equipe que presta o cuidado dentro das ILPI.

Salienta-se, portanto, que mesmo que o idoso seja independente, quando este passa a residir em ILPI, "pode desenvolver diferentes níveis de dependência, evento relacionado à sua dificuldade de aceitação e de adaptação às novas condições de vida, bem como à falta de motivação e de encorajamento"(20:616) que são situações comuns nas ILPI.

Com relação ao perfil e perda cognitiva, uma recente pesquisa ${ }^{(21)}$ realizada com idosas longevas de Fortaleza-CE apresentou maior perda em "atenção e cálculo" bem como nos domínios que estão relacionados com as habilidades adquiridas no ambiente escolar, tais como "linguagem", para o qual são exigidos requisitos como leitura, escrita, atenção e cálculo. O estudo ${ }^{(21)}$ também demonstrou que o nível de escolaridade também influencia no déficit cognitivo, uma vez que as maiores perdas estavam entre as idosas com menor grau de escolaridade ou analfabetas.

Destaca-se também que algumas habilidades cognitivas como o vocabulário, tem maior resistência ao envelhecimento do cérebro, podendo até mesmo aprimorar com a idade. Todavia, outras habilidades, como o raciocínio conceitual, memória e velocidade de processamento, reduzem gradualmente ao longo do tempo ${ }^{(7)}$.

Nesse sentido, outra pesquisa ${ }^{(22)}$ desenvolvida com idosos no Estado do Maranhão que apresentavam declínio cognitivo, demonstrou que 57 (45,83\%) tinham baixa escolaridade, o que ratifica não só os resultados encontrados no presente estudo, como também a relação de escores do rastreio cognitivo proposto pelo MEEM.

Já em relação ao sexo, um estudo ${ }^{(8)}$ sobre perfil cognitivo com 60 idosos residentes em ILPI mostrou que a população idosa feminina tem maior expectativa de vida, porém maior predisposição a desenvolver demências, sendo a doença de Alzheimer a de maior incidência. Constatou também que $18(30 \%)$ dos idosos possuíam perda cognitiva a partir da classificação do MEEM, sendo que essa perda cognitiva foi maior no sexo feminino.

Outra pesquisa ${ }^{(23)}$ realizada com 503 idosos assistidos por Equipes Saúde da Família de DouradosMS também encontrou correlação significativa entre o déficit cognitivo associado ao gênero feminino, acometendo cerca de $230(45,8 \%)$ da população estudada.

A função cognitiva é um fator importante fator para avaliar a qualidade de vida de idosos, e a sua disfunção está relacionada a aspectos individuais e também sociais(22). E também "é um dos determinantes da qualidade de vida na velhice, pois perdas nas funções cognitivas podem resultar em prejuízo no funcionamento físico, social e emocional de idosos" (24:157). Dessa forma, a partir dessas referências é possível inferir que as alterações na função cognitiva podem interferir negativamente na vida dos idosos, tanto no desenvolvimento de atividades cotidianas como na sua qualidade de vida.

Como limitação do estudo, não foi possível aumentar o tamanho da amostra, uma vez que nas ILPI pesquisadas o número de longevos que atendiam aos critérios de inclusão foi pequeno. Associado a isso, houve dificuldades em encontrar estudos recentes nesta perspectiva e que avaliam idosos longevos.

\section{CONSIDERAÇÕES FINAIS}

O estudo permitiu avaliar a dependência funcional para as ABVD a partir de dois instrumentos, o que nos faz inferir que no contexto das duas ILPI pesquisadas os longevos apresentam graus diferenciados de dependência. Esta realidade requer maior cuidado e preocupação por parte das ILPI quanto à manutenção das ABVD, pois a capacidade para executar as atividades diárias é um indicador da funcionalidade dos idosos. Com relação à função cognitiva, os longevos apresentaram médias baixas em quase todos os itens do MEEM. O estudo também demonstrou que houve maior perda cognitiva nos indivíduos do sexo feminino e naqueles que não são alfabetizados.

Os conhecimentos daqui advindos também podem suscitar nas ILPI, e também na sociedade em geral, uma discussão a partir do impacto que a dependência funcional traz para a saúde do idoso, 
fomentando o debate sobre políticas públicas destinadas ao idoso, principalmente aqueles que residem em ILPI. Assim, novos planos de intervenção podem ser estabelecidos, para melhoria nas ABVD e nas AIVD, tanto para os longevos dependentes funcionalmente como para aqueles independentes.

\section{AGRADECIMENTOS}

Aos longevos que permitiram que pudéssemos avaliá-los, demonstrando confiança em nosso trabalho. As ILPI pela autorização do local de pesquisa e em especial aos idosos que participaram deste estudo.

\section{REFERÊNCIAS}

1. Instituto Brasileiro de Geografia e Estatística (IBGE). Projeção da população do Brasil e das Unidades da Federação. [Internet] 2016 [acesso em 16 jul 2016]. Disponível: http://www.ibge.gov.br/apps/populacao/projecao/

2. Closs VE, Schwanke CHA. A evolução do índice de envelhecimento no Brasil, nas suas regiões e unidades federativas no período de 1970 a 2010. Rev. bras. geriatr. gerontol. [Internet] 2012;15(3) [acesso em 13 nov 2016]. Disponível: http://dx.doi.org/10.1590/S1809-98232012000300006

3. Instituto Brasileiro de Geografia e Estatística (IBGE). Sinopse do Censo Demográfico 2010. Rio de janeiro. [internet] 2011 [acesso em08 jul 2016]. Disponível: http://biblioteca.ibge.gov.br/visualizacao/livros/liv49230.pdf

4. Fuhrmann AC, Bierhals CCBK, dos Santos NO, Paskulin LMG.Associação entre a capacidade funcional de idosos dependentes e a sobrecarga do cuidador familiar. Rev. gauch. enferm. [Internet] 2015;36(1) [acesso em 15 nov 2016]. Disponível: http://www.lume.ufrgs.br/handle/10183/117452

5. Lopes GL, Santos MIPO. Funcionalidade de idosos cadastrados em uma unidade da Estratégia Saúde da Família segundo categorias da Classificação Internacional de Funcionalidade. Rev. bras. geriatr. gerontol. [Internet] 2015;18(1) [acesso em 16 nov. 16]. Disponível: http://dx.doi.org/10.1590/1809-9823.2015.14013

6. dosReis LA, Moreira JSS, Duarte SFP. Risco de queda, capacidade funcional e respiratória em idosos residentes em instituições de longa permanência.Rev Neurocienc. [Internet] 2015;23(3) [acesso em 08 ago 2016]. Disponível: http://dx.doi.org/10.4181/RNC.2015.23.03.1071.08p

7. Harada CN, Love MCN, Triebel KL. Normal Cognitive Aging. Clin Geriatr Med. [Internet] 2013;29(4) [acesso em 25 jul 2016]. Disponível: http://dx.doi.org/10.1016/j.cger.2013.07.002

8. Ferreira LS, Pinho MSP, Pereira MWM, Ferreira AP. Perfil cognitivo de idosos residentes em Instituições de Longa Permanência de Brasília-DF. Rev. bras. enferm. [Internet] 2014;67(2) [acesso em 11 jul 2016]. Disponível: http://dx.doi.org/10.5935/0034-7167.20140033

9. Coping With Mild Cognitive Impairment.The New York Times [Internet] New York; 2012. [acesso em11 jul 2016 ]. Disponível: http://newoldage.blogs.nytimes.com/2012/09/10/coping-with-mild-cognitive-impairment/?_r=0

10. Fleck CS, Gerzson LR, Steidl SEM, Hernandez NM. Caracterização da capacidade funcional, nível cognitivo e força muscular respiratória de idosas com síndrome parkinsoniana. Estud. interdiscipl. envelhec. [Internet] 2014;19(1) [acesso em 16 nov 2016]. Disponível: http://seer.ufrgs.br/index.php/RevEnvelhecer/article/ download/40548/31004

11. Bentes ACO, Pedroso JS, Maciel CAB. O idoso nas instituições de longa permanência: uma revisão bibliográfica. Aletheia [Internet]. 2012;38-39 [acesso em 19 nov 2016]. Disponível:http://pepsic.bvsalud.org/scielo. php?script=sci_arttext\&pid=S1413-03942012000200016\&lng=pt.

12. Estivalet KM, Palma KAXA. Estimulação de memória em Instituição de Longa Permanência para idosos. Rev. Neurocienc. [Internet] 2014;22(3) [acesso em22jul 2016]. Disponível: http://dx.doi.org/10.4181/ RNC.2014.22.03.950.8p

13. MarinhoLM, Vieira MA, Costa SM, Andrade JMO. Grau de dependência de idosos residentes em instituições de longa permanência. Rev. gauch. enferm. [Internet] 2013;34(1) [acesso em 12 jul 2016]. Disponível: http://dx.doi. 
14. Ministério da Saúde (BR). Secretaria de Atenção à Saúde. Departamento de Atenção Básica. Caderno de Atenção Básica n.19. Envelhecimento e Saúde da pessoa idosa. Brasília (DF): Ministério da Saúde; 2007.

15. Rempel C, Haetinger C, Sehnem E. Reflexões de idosos sobre as relações entre o trabalho rural, problemas de coluna e postura corporal. Estud. Soc. e Agric. [Internet] 2013;21(2) [acesso em 19 nov 2016]. Disponível: http:// r1.ufrrj.br/esa/V2/ojs/index.php/esa/article/viewFile/369/365

16. Vaz SFA, Gaspar NMS. Depressão em idosos institucionalizados no distrito de Bragança. Rev Enferm. [Internet] 2011;3(4) [acesso em 18 jul 2016]. Disponível: https://repositorio-aberto.up.pt/bitstream/10216/57264/2/87104.pdf

17. Duarte MR, Santos MLT, Batista MFG, Moura P. Efeitos do processo do envelhecimento na capacidade funcional dos idosos e suas formas de mensuração. EFDeportes.com, Revista Digital. [Internet] 2014;19 [acesso em 19 nov 2016]. Disponível: http://www.efdeportes.com/efd192/capacidade-funcional-dos-idosos-e-mensuracao.htm

18. Lenardt MH, Carneiro NHK. Associação entre as características sociodemográficas e a capacidade funcional de idosos longevos da comunidade. Cogitare Enferm. [Internet] 2013;18(1) [acesso em 30 set 2016]. Disponível:http:// dx.doi.org/10.5380/ce.v18i1.31299

19. Pereira FM; Besse M. Fatores associados à independência funcional de idosos residentes em instituição de longa permanência. Acta Fisiátr. [Internet] 2011;18(2) [acesso em 18 jul 2016]. Disponível: http://www.actafisiatrica. org.br/detalhe_artigo.asp?id=80

20. Feliciani AM, Santos SSC, Valcarenghi RV. Funcionalidade e quedas em idosos institucionalizados: Propostas de ações de enfermagem. Cogitare Enferm. [Internet] 2011;16(4) [acesso em 01 out 2016]. Disponível: http:// dx.doi.org/10.5380/ce.v16i4.22456

21. Domiciano BR, Braga DKAP, da Silva PN, de Vasconcelos TB, Macena RHM. Escolaridade, idade e perdas cognitivas de idosas residentes em instituições de longa permanência. Rev Neurocienc. [Internet] 2014;22(3) [acesso em 02 jul 2016]. Disponível: http://dx.doi.org/10.4181/RNC.2014.22.03.971.7p

22. Chaves AS, dos Santos AM, Alves MTSSB, Salgado Filho N. Associação entre declínio cognitivo e qualidade de vida de idosos hipertensos. Rev. bras. geriatr. gerontol. [Internet] 2015;18(3) [acesso em 08 ago 2016]. Disponível: http://dx.doi.org/10.1590/1809-9823.2015.14043

23. Santos CS, Cerchiari EAN, Alvarenga MRM, Faccenda O, Oliveira MAC. Avaliação da confiabilidade do MiniExame do Estado Mental em idosos e associação com variáveis sociodemográficas. Cogitare Enferm. [Internet] 2010;15(3) [acesso em 01 out 2016]. Disponível: http://dx.doi.org/10.5380/ce.v15i3.18879

24. Beckert M, Irigaray TQ, Trentini CM. Qualidade de vida, cognição e desempenho nas funções executivas de idosos. Estud. psicol. (Campinas). [Internet] 2012;29(2) [acesso em 17 nov 2016]. Disponível: http://dx.doi. org/10.1590/S0103-166X2012000200001 